Pacific

Journal of

Mathematics

SPRINGER THEORY IN BRAID GROUPS AND THE BIRMAN-KO-LEE MONOID

David Bessis, François Digne, and Jean Michel 


\title{
SPRINGER THEORY IN BRAID GROUPS AND THE BIRMAN-KO-LEE MONOID
}

\author{
David Bessis, François Digne, and Jean Michel
}

\begin{abstract}
We state a conjecture about centralizers of certain roots of central elements in braid groups, and check it for braid groups of type $A, B, G(d, 1, r)$ and a couple of other cases. Our proof makes use of results from Birman-Ko-Lee, of which we give a new intrinsic account.
\end{abstract}

Notations. If $G$ is a group acting on a set $X$, we denote by $X^{G}$ the subset of $X$ of elements fixed by all elements of $G$. If $(X, x)$ is a pointed topological space, we denote by $\Omega(X, x)$ the corresponding loop space, by $\sim$ the homotopy relation on $\Omega(X, x)$ and by $\pi_{1}(X, x)$ the fundamental group. For all $n \in \mathbf{N}$, we denote by $\mu_{n}$ the set of $n$-th roots of unity in $\mathbf{C}$.

\section{Introduction.}

Springer theory of regular elements (introduced in $[\mathbf{S p}]$ ) explains how certain complex reflection groups naturally arise as centralizers in other complex reflection groups of particular elements, the regular elements. The construction relies on invariant theory and elementary algebraic geometry, and gives precise information on the relation between the two reflection groups involved (in terms of basic invariants, hyperplane arrangements,...). In their work on Deligne-Lusztig varieties $([\mathbf{B r M i}])$, Broué and the third author gave a partial interpretation of Springer theory in terms of roots of central elements in braid groups associated to complex reflection groups. Following some of the implicit ideas in [BrMi], we state (and prove in some cases) a conjecture which explains how Springer theory of regular elements can be seen as the image in complex reflection groups of a more general theory holding in braid groups. Of course, Springer's original invariant-theoretical arguments have no analog in braid groups. Instead, we rely on properties of the monoid structure recently discovered by Birman-Ko-Lee ([BiKoLee]) and on a geometric interpretation of this monoid.

Before stating our conjecture, let us recall some properties of complex reflection groups. Let $V$ be a finite dimensional complex vector space and let $W \subset \mathrm{GL}(V)$ be an irreducible finite group generated by complex reflections (that is, elements $s \in \mathrm{GL}(V)$ such that $\operatorname{ker}(s-\mathrm{Id})$ is a hyperplane). We denote by $\mathcal{M}$ the complement in $V$ of the union of all reflecting hyperplanes. 
The elements of $\mathcal{M}$ are said to be regular vectors. The space $\mathcal{M} / W$ is called the complement of the discriminant of $W$. Choose a basepoint in $\mathcal{M}$, take its image as basepoint for $\mathcal{M} / W$. The braid group $\mathbf{B}_{W}$ associated to $W$ is, by definition, the fundamental group of $\mathcal{M} / W$. The fundamental group of $\mathcal{M}$ is the pure braid group, denoted by $\mathbf{P}_{W}$. The covering $\mathcal{M} \rightarrow \mathcal{M} / W$ is unramified and one has an exact sequence

$$
1 \longrightarrow \mathbf{P}_{W} \longrightarrow \mathbf{B}_{W} \longrightarrow W \longrightarrow 1 \text {. }
$$

Let $\zeta \in \mathbf{C}$ be a root of unity of order $d$. An element $w \in W$ is $\zeta$-regular, as defined in $[\mathbf{S p}]$, if and only if it admits an regular eigenvector with eigenvalue $\zeta$ or, in other words, if and only if

$$
\operatorname{ker}(w-\zeta \mathrm{Id}) \cap \mathcal{M} \neq \emptyset .
$$

Assume that there exists a $\zeta$-regular element $w(\zeta$ is then said to be a regular eigenvalue, its order $d$ is a regular number $)$. Let $V(w):=\operatorname{ker}(w-\zeta \mathrm{Id})$ and $\mathcal{M}(w):=\operatorname{ker}(w-\zeta \mathrm{Id}) \cap \mathcal{M}$. The centralizer $C_{W}(w)$ stabilizes each eigenspace of $w$. In particular, $C_{W}(w)$ has a linear representation in $V(w)$. According to the main result in $[\mathbf{S p}]$, this representation is faithful and $C_{W}(w)$, viewed as a linear group acting on $V(w)$, is a complex reflection group. Moreover, the set of regular vectors for this reflection group is precisely $\mathcal{M}(w)$. Let $\mathbf{B}_{C_{W}(w)}$ be the braid group associated with $C_{W}(w)$, i.e., the fundamental group of $\mathcal{M}(w) / C_{W}(w)$.

Let $k$ be such that $\zeta=e^{2 i \pi k / d}$. Assume that the basepoint $x_{0} \in \mathcal{M}$ is chosen in $\mathcal{M}(w)$. Let $\gamma$ be the path:

$$
\begin{aligned}
\gamma:[0,1] & \longrightarrow \mathcal{M} \\
t & \longmapsto e^{2 i \pi k t / d} x_{0} .
\end{aligned}
$$

Let $p$ be the quotient map $\mathcal{M} \rightarrow \mathcal{M} / W$. As noticed in [BrMi] (p. 92), the composed map $p \circ \gamma$ is a loop in $\mathcal{M} / W$, defining an element $\mathbf{w} \in \mathbf{B}_{W}$, such that $\mathbf{w}^{d}$ is central in $\mathbf{B}_{W}$, and $w$ is the image of $\mathbf{w}$ by the canonical epimorphism $\mathbf{B}_{W} \rightarrow W$. In other words, there is a natural way of lifting a regular element of $W$ to a root of a central element in $\mathbf{B}_{W}$. Moreover, the natural map $\mathcal{M}(w) / C_{W}(w) \rightarrow \mathcal{M} / W$ defines a morphism $\mathbf{B}_{C_{W}(w)} \rightarrow \mathbf{B}_{W}$, whose image can be easily checked to lie in the centralizer $C_{\mathbf{B}_{W}}(\mathbf{w})$.

The following conjecture claims that there is an analog of Springer theory in braid groups, where the regular elements have to be replaced by the corresponding roots of central elements:

Conjecture 0.1. The natural morphism $\mathbf{B}_{C_{W}(w)} \rightarrow \mathbf{B}_{W}$ induces an isomorphism between $\mathbf{B}_{C_{W}(w)}$ and the centralizer $C_{\mathbf{B}_{W}}(\mathbf{w})$.

The conjecture can be reformulated in a more intrinsic way:

Let $(X, x)$ be a pointed topological space and $G$ be a group acting on $(X, x)$ (by morphisms in the category of pointed spaces, so $\left.x \in X^{G}\right)$. The 
action of $G$ can be naturally extended to the loop space $\Omega(X, x)$. As clearly $\forall g \in G, \forall \gamma, \gamma^{\prime} \in \Omega(X, x), \gamma \sim \gamma^{\prime} \Leftrightarrow g(\gamma) \sim g\left(\gamma^{\prime}\right)$, this induces a morphism $G \rightarrow \operatorname{Aut}\left(\pi_{1}(X, x)\right)$. For a given $G$, the construction of $\pi_{1}(X, x)^{G}$ from $(X, x)$ is actually functorial, from the category of pointed topological spaces with $G$-action to the category of groups. Thus the natural injection $\left(X^{G}, x\right) \subseteq(X, x)$ induces a natural morphism from $\pi_{1}\left(X^{G}, x\right)=\pi_{1}\left(X^{G}, x\right)^{G}$ to $\pi_{1}(X, x)^{G}$.

If $W$ is as above a finite irreducible complex reflection group in $\mathrm{GL}(V)$, we denote by $X$ the corresponding space $\mathcal{M} / W$. For all integers $m$, the inclusion $\mu_{m} \subset \mathbf{C}^{\times}$and the identification of $\mathbf{C}^{\times}$with the center of $\operatorname{GL}(V)$ define a natural quotient action of $\mu_{m}$ on $X$. As noticed in [Be] 1.2, when $d$ is regular and $w$ and $\mathbf{w}$ are as defined above, $\mathcal{M}(w) / C_{W}(w)$ is homeomorphic to its image $X^{\mu_{d}}$ in $X$. Let $x \in X^{\mu_{d}}$. It is an easy calculation to check that the subgroup of $\operatorname{Aut}\left(\pi_{1}(X, x)\right)$ generated by the conjugation by $\mathbf{w}$ coincides with the one arising from the action of $\mu_{d}$ on $X$.

Thus the above conjecture is equivalent to the statement that the natural morphism

$$
\pi_{1}\left(X^{\mu_{d}}, x\right) \rightarrow \pi_{1}(X, x)^{\mu_{d}}
$$

is an isomorphism.

The fact that the morphism is an isomorphism doesn't depend on the choice of $x \in X^{\mu_{d}}$.

Our main theorem checks the conjecture for some specific groups (we use the standard notations from $[\mathbf{S h T o}])$ :

Theorem 0.2. Let $W$ be an irreducible complex reflection group. Suppose $W$ is of one of the following types: $\mathfrak{S}_{n} ; G(p, 1, r)$ with $p>1 ; G_{4} ; G_{5} ; G_{8}$; $G_{10} ; G_{16} ; G_{18} ; G_{25} ; G_{26} ; G_{32}$. Let $X$ be the complement of the discriminant of $W$. Let $d$ be a regular number for $W$, and let $x \in X^{\mu_{d}}$. The natural morphism

$$
\pi_{1}\left(X^{\mu_{d}}, x\right) \rightarrow \pi_{1}(X, x)^{\mu_{d}}
$$

is an isomorphism.

\section{The local monoid.}

We will deduce our theorem from the particular case where $W$ is the symmetric group $\mathfrak{S}_{n}$, and $\mathbf{B}$ the Artin braid group on $n$ strings. In [BiKoLee], Birman, Ko and Lee describe a remarkable monoid for this group. The properties of their monoid will be crucial in our proof. However, contrary to what is done in [BiKoLee], where the Artin braid group is initially given by the Artin presentation, we prefer to use its more intrinsic definition as a 
fundamental group. Of course, both viewpoints give (non-canonically) isomorphic groups; however, we believe our reformulation is more natural than the original description. ${ }^{1}$

Let $X_{n}$ be the space of subsets of $\mathbf{C}$ of cardinal $n$, with its natural topology. In the setting of the introduction, when taking the natural irreducible reflection representation of the symmetric group $W=\mathfrak{S}_{n}$ on $\mathbf{C}^{n-1}$, the complement of the discriminant $\mathcal{M} / W$ is homotopy equivalent (in a way compatible with the action of $\left.\mathbf{C}^{\times}\right)$to $X_{n}$. We choose the usual direct (= anti-clockwise) orientation on $\mathbf{C}$.

Let us choose a basepoint $x \in X_{n}$, and let $B_{x}=\pi_{1}\left(X_{n}, x\right)$. We define in this section a monoid $M_{x}$, which is a set of group generators for $B_{x}$. The structure of $M_{x}$ depends on the choice of $x$ : When $x$ is taken to be the "usual" basepoint $\{1, \ldots, n\}$, the monoid $M_{x}$ will be isomorphic to the usual Artin monoid; choosing $\mu_{n}$ (the set of $n$-roots of unity) will yield what we call the Birman-Ko-Lee monoid, which is isomorphic to the one described in [BiKoLee].

If $\gamma \in \Omega\left(X_{n}, x\right)$ and $z \in x$, we denote by $\gamma_{z}$ the "string" of $\gamma$ with origin $z$. It is a path $[0,1] \rightarrow \mathbf{C}$, with $\gamma_{z}(0)=z$ and $\gamma_{z}(1) \in x$. The element $\gamma$ is uniquely determined by $\left(\gamma_{z}\right)_{z \in x}$. Conversely, a set of $n$ such strings which do not intersect define an element of $\Omega\left(X_{n}, x\right)$.

Definition 1.1. A pair $\left\{z, z^{\prime}\right\} \subset x$ is said to be non-crossing if and only if the closed line segment $\left[z, z^{\prime}\right]$ intersects $x$ only at $z$ and $z^{\prime}$.

We denote by $S_{x}$ the set of non-crossing pairs of elements of $x$.

Examples. The two crucial examples are $x=\{1, \ldots, n\}$ and $x=\mu_{n}$. The corresponding $S_{x}$ have respectively cardinal $n-1$ and $n(n-1) / 2$.

For each $\left\{z, z^{\prime}\right\} \in S_{x}$, we denote by $\delta_{\left\{z, z^{\prime}\right\}} \in B_{x}$ the generator of the monodromy naturally associated to $\left[z, z^{\prime}\right]$, as in the appendix of $[\mathbf{B e}]$. A representative of this element can be defined for instance by the set of strings $\gamma_{z^{\prime \prime}}(t)=z^{\prime \prime}$ if $z^{\prime \prime} \notin\left\{z, z^{\prime}\right\}, \gamma_{z}(t)=f\left(z, z^{\prime}, \epsilon\right)(t), \gamma_{z^{\prime}}(t)=f\left(z^{\prime}, z, \epsilon\right)(t)$ for $\epsilon \in \mathbf{R}_{+}^{*}$ small enough (depending only on $x$ ) where

$$
\forall t, f\left(z, z^{\prime}, \epsilon\right)(t)=\frac{z+z^{\prime}}{2}+\frac{z-z^{\prime}}{2} \cos (\pi t)+i \epsilon \frac{z-z^{\prime}}{2} \sin (\pi t)
$$

(the corresponding arc is a half-ellipse with great axis $\left[z, z^{\prime}\right]$ and small axis of length $\epsilon\left|z-z^{\prime}\right|$. The exact choice of $f\left(z, z^{\prime}, \epsilon\right)$ is not important. One could for example replace the half-ellipse by a half-rhomb, or any other variation. However, for later use where defining a loop up to homotopy will not be sufficient, it is convenient for us to define this explicit element of $\left.\Omega\left(X_{n}, x\right)\right)$.

${ }^{1}$ After the present article was accepted for publication, the authors were informed that T. Brady had independently obtained a similar description of the Birman-Ko-Lee monoid. 
Definition 1.2. The submonoid $M_{x}$ of $B_{x}$ generated by

$$
\left\{\delta_{\left\{z, z^{\prime}\right\}} \mid\left\{z, z^{\prime}\right\} \in S_{x}\right\}
$$

is called the local monoid at $x$.

Clearly, the map $\left\{z, z^{\prime}\right\} \mapsto \delta_{\left\{z, z^{\prime}\right\}}$ is injective, so we may identify $S_{x}$ with its image in $M_{x}$. Let $l$ be the natural length function on $B_{x}$ (the map $B_{x} \rightarrow \mathbf{Z}$ induced by the discriminant function $\mathcal{M} \rightarrow \mathbf{C}^{\times}$). The elements of $S_{x}$, being generators of the monodromy, have length 1 , so the monoid $M_{x}$ is $\mathbf{N}$-graded (only the trivial element being of length 0 ).

We denote by $\prec$ the left divisibility relation in $M_{x}$, i.e.,

$$
\forall m, m^{\prime} \in M_{x}, m \prec m^{\prime} \Leftrightarrow \exists m^{\prime \prime} \in M_{x}, m m^{\prime \prime}=m^{\prime} .
$$

It results from the $\mathbf{N}$-grading of $M_{x}$ that the relation $\prec$ is a partial order on $M_{x}$.

We will later study extensively the structure of $M_{\mu_{n}}$. We complete this section with some first properties which are valid for all $x$.

Proposition 1.3. The set $S_{x}$ is a set of group generators for $B_{x}$ (i.e., by taking $S_{x} \cup\left\{s^{-1} \mid s \in S_{x}\right\}$, one has a set of monoid generators for $\left.B_{x}\right)$.

Proof. Distinguish one point $z \in x$. Draw the segments $\left\{\left[z, z^{\prime}\right] \mid z^{\prime} \in x\right\}$. Some of them may be crossing, but by splitting these into smaller ones, one gets a planar graph connecting all points in $x$ and whose edges are noncrossing. The result then follows from the main theorem in [Se] and its reformulation in the appendix of $[\mathbf{B e}]$.

Notations and conventions. We write $\lambda \vdash x$ to say that $\lambda$ is a partition of $x$, in the usual set theoretical sense. If $y \subset x$ and $\lambda \vdash y$, we will also use $\lambda$ to denote the partition of $x$ obtained by completing $\lambda$ with parts of cardinal 1. In other words, we will sometimes, for convenience, omit to write the cardinal 1 parts of a partition. By convex polygon, we mean either a point (if the number of vertices is 1 ), a segment (if the number of vertices is 2), or (if the number of vertices is 3 or more) a nondegenerate convex polygon, i.e., such that three vertices never lie on the same line. When $y \subset \mathbf{C}$, we write $\bar{y}$ for the convex hull of $y$.

We now extend the notion of non-crossedness to partitions of $x$ :

\section{Definition 1.4.}

- A finite nonempty subset $y \subset \mathbf{C}$ is said to be convex if and only if it is the set of vertices of a convex polygon in $\mathbf{C}$.

- A partition $\lambda$ of $x$ is said to be non-crossing if and only if it satisfies the following two properties:

- Every part $\nu$ in $\lambda$ is convex.

- If two parts $\nu_{1}$ and $\nu_{2}$ are distinct, their convex hulls $\overline{\nu_{1}}$ and $\overline{\nu_{2}}$ do not intersect. 
- The set of non-crossing partitions of $x$ is denoted by $\mathcal{P}_{x}$. We use the notation $\lambda=x$ to express that $\lambda \in \mathcal{P}_{x}$.

We already have a notion of non-crossing pairs. The partition $\left\{\left\{z, z^{\prime}\right\}\right\}$ is non-crossing if and only if $\left\{z, z^{\prime}\right\}$ is a non-crossing pair.

Let $\nu$ be a part of a non-crossing partition of $x$. Choose $z_{1}$ an arbitrary element of $\nu$. For $i \in\{1, \ldots, k\}$, denote by $z_{i}$ the $i$-th element of $\nu$ for the clockwise order on $\nu$ starting at $z_{1}$. In the next lemma, when $k=1$, the product is the empty product, thus the trivial element in $M_{x}$.

Lemma 1.5. The element $\delta_{\left\{z_{1}, z_{2}\right\}} \delta_{\left\{z_{2}, z_{3}\right\}} \ldots \delta_{\left\{z_{k-2}, z_{k-1}\right\}} \delta_{\left\{z_{k-1}, z_{k}\right\}} \in M_{x}$ does not depend on the choice of $z_{1}$ in $\nu$.

Proof. This is a consequence of one of the Sergiescu relations (see [Se], 1.1(ii) or $[\mathbf{B e}]$, Théorème A.6) namely that

$$
\begin{aligned}
\delta_{\left\{z_{1}, z_{2}\right\}} & \delta_{\left\{z_{2}, z_{3}\right\}} \cdots \delta_{\left\{z_{k-2}, z_{k-1}\right\}} \delta_{\left\{z_{k-1}, z_{k}\right\}} \\
= & \delta_{\left\{z_{k}, z_{1}\right\}} \delta_{\left\{z_{1}, z_{2}\right\}} \delta_{\left\{z_{2}, z_{3}\right\}} \cdots \delta_{\left\{z_{k-2}, z_{k-1}\right\}}
\end{aligned}
$$

The proof of this relation is by induction on $k$. The case $k=3$ is checked by a direct computation, and for other $k$ we have

$$
\begin{aligned}
\delta_{\left\{z_{1}, z_{2}\right\}} & \delta_{\left\{z_{2}, z_{3}\right\}} \ldots \delta_{\left\{z_{k-2}, z_{k-1}\right\}} \delta_{\left\{z_{k-1}, z_{k}\right\}} \\
& =\delta_{\left\{z_{2}, z_{3}\right\}} \cdots \delta_{\left\{z_{k-2}, z_{k-1}\right\}} \delta_{\left\{z_{k-1}, z_{1}\right\}} \delta_{\left\{z_{k-1}, z_{k}\right\}} \\
& =\delta_{\left\{z_{2}, z_{3}\right\}} \cdots \delta_{\left\{z_{k-2}, z_{k-1}\right\}} \delta_{\left\{z_{k-1}, z_{k}\right\}} \delta_{\left\{z_{k}, z_{1}\right\}}
\end{aligned}
$$

where the first equality is by induction and the second by the case $k=3$.

We denote $\delta_{\nu}$ the element of 1.5 (when $\left\{z, z^{\prime}\right\}$ is a non-crossing pair, $\delta_{\left\{\left\{z, z^{\prime}\right\}\right\}}$ coincides with the element $\delta_{\left\{z, z^{\prime}\right\}}$ defined earlier).

Lemma 1.6. Let $\nu$ and $\nu^{\prime}$ be two finite nonempty subsets of $\mathbf{C}$. Suppose $\left\{\nu, \nu^{\prime}\right\}$ can be completed to a non-crossing partition of $x$. Then we have $\delta_{\nu} \delta_{\nu^{\prime}}=\delta_{\nu^{\prime}} \delta_{\nu}$.

Proof. The convex hulls of $\nu$ and $\nu^{\prime}$ cannot intersect, thus the generators corresponding to their edges commute pairwise.

Let $\lambda \models x$. The above lemma makes it natural (and non-ambiguous) to define

$$
\delta_{\lambda}=\prod_{\nu \in \lambda} \delta_{\nu}
$$

Definition 1.7. Given two partitions $\lambda$ and $\lambda^{\prime}$ of $x$, we say that $\lambda$ is finer than $\lambda^{\prime}$ (or equivalently that $\lambda^{\prime}$ is coarser than $\lambda$ ), and we write $\lambda \prec \lambda^{\prime}$, if and only if $\forall \nu \in \lambda, \exists \nu^{\prime} \in \lambda^{\prime}, \nu \subset \nu^{\prime}$.

Clearly $\prec$ is a partial order on the set of partitions of $x$. We intentionally use the same symbol $\prec$, as for the left divisibility order in $M_{x}$. The next proposition justifies this notation. 
Let $P_{x}=\left\{\delta_{\lambda} \mid \lambda \in \mathcal{P}_{x}\right\}$. The main result in this section is the following proposition.

\section{Proposition 1.8.}

(i) The left divisibility order in $P_{x}$ coincides with the restriction of $\prec$ from $M_{x}$ to $P_{x}$; in other words,

$$
\forall p, p^{\prime} \in P_{x},\left(\exists p^{\prime \prime} \in P_{x}, p p^{\prime \prime}=p^{\prime}\right) \Leftrightarrow\left(\exists m^{\prime \prime} \in M_{x}, p m^{\prime \prime}=p^{\prime}\right) .
$$

We denote this partial order by $\prec$.

(ii) The map

$$
\begin{aligned}
D: \mathcal{P}_{x} & \longrightarrow P_{x} \\
\lambda & \longmapsto \delta_{\lambda}
\end{aligned}
$$

is a poset isomorphism from $\left(\mathcal{P}_{x}, \prec\right)$ to $\left(P_{x}, \prec\right)$.

Before proving the proposition, we need some definitions and lemmas.

Definition 1.9. Let $\nu$ be a convex subset of $\mathbf{C}$, and let $\nu^{\prime}$ be a subset of $\nu$. Let $z_{1}, \ldots, z_{k}$ be a clockwise numbering of the elements of $\nu$, such that $z_{i_{1}}, \ldots, z_{i_{k^{\prime}}}$ is a clockwise numbering of $\nu^{\prime}$ with $1=i_{1}<i_{2}<\cdots<i_{k^{\prime}} \leq$ $k$. Then we denote by $\nu^{\prime} \backslash \nu\left(\nu\right.$ "cut at" $\left.\nu^{\prime}\right)$ the partition with parts the sets $\left\{z_{i_{j}}, z_{i_{j}+1}, \ldots, z_{i_{j+1}-1}\right\}$ (where for $j=k^{\prime}$ we make the convention that $\left.i_{k^{\prime}+1}=k+1\right)$.

Example. In the picture below, the grey points are the points of $\nu^{\prime}$, the other points of $\nu$ are black, and the parts of $\nu^{\prime} \backslash \nu$ are enclosed by dashed curves.

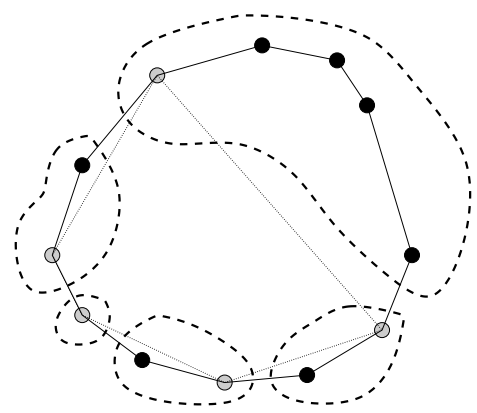

Note that $\nu^{\prime} \backslash \nu$ is well-defined (does not depend on the chosen numbering) and is a non-crossing partition of $\nu$. We will need the following alternative description of $\nu^{\prime} \backslash \nu$ : Its parts of cardinal $\geq 2$ are the intersections of $\nu$ with each connected component of the complement of $\overline{\nu^{\prime}}$ in $\bar{\nu}$, to which has been added the element of $\nu^{\prime}$ just before the connected component (in clockwise order). 
Denote by $\phi_{x}$ the natural epimorphism from $B_{x}$ to $\mathfrak{S}_{x}$. It maps $\delta_{\left\{z, z^{\prime}\right\}}$ to the transposition $\left(z, z^{\prime}\right)$. Thus, when $\lambda \models x$, it is clear by construction that $\lambda$ is the orbit decomposition for the action in $x$ of $\phi_{x}\left(\delta_{\lambda}\right)$. Thus if $\lambda \neq \lambda^{\prime}$, then $\phi_{x}\left(\delta_{\lambda}\right)$ and $\phi_{x}\left(\delta_{\lambda^{\prime}}\right)$ have different cycle decompositions, and are different. We have proved the:

Lemma 1.10. The restriction of $\phi_{x}$ to $P_{x}$ is injective.

\section{Lemma 1.11.}

(i) Let $\nu$ be a convex subset of $\mathbf{C}$, and let $\nu^{\prime}$ be a subset of $\nu$. We have $\delta_{\nu}=\delta_{\nu^{\prime}} \delta_{\nu^{\prime} \backslash \nu}$.

(ii) Suppose $\lambda, \lambda^{\prime} \models x, \lambda^{\prime}$ has only one part of cardinal $\geq 2$, and $\lambda$ is finer that $\lambda^{\prime}$. Then there exists a unique non-crossing partition of $x$, which we denote by $\lambda \backslash \lambda^{\prime}$ such that $\delta_{\lambda} \delta_{\lambda \backslash \lambda^{\prime}}=\delta_{\lambda^{\prime}}$.

(iii) Suppose $\lambda, \lambda^{\prime} \models x$ and $\lambda$ is finer that $\lambda^{\prime}$. Then there exists a unique non-crossing partition of $x$, which we denote by $\lambda \backslash \lambda^{\prime}$ such that $\delta_{\lambda} \delta_{\lambda \backslash \lambda^{\prime}}=$ $\delta_{\lambda^{\prime}}$.

Proof. We prove (i) by induction on the cardinality of $\nu^{\prime}$. With the notations of 1.9 , let $\nu_{1}=\left\{z_{1}, \ldots, z_{i_{2}-1}\right\}, \nu_{2}=\nu-\nu_{1}$ so that $\delta_{\nu}=\delta_{\nu_{1}} \delta_{\left\{z_{i_{2}-1}, z_{i_{2}}\right\}} \delta_{\nu_{2}}$, and let $\nu_{2}^{\prime}=\nu^{\prime}-\left\{z_{1}\right\}$, so that $\delta_{\nu^{\prime}}=\delta_{\left\{z_{1}, z_{i_{2}}\right\}} \delta_{\nu_{2}^{\prime}}$. Then by 1.5 applied to $\nu_{1} \cup\left\{z_{i_{2}}\right\}$ we have $\delta_{\nu}=\delta_{\left\{z_{1}, z_{2}\right\}} \delta_{\nu_{1}} \delta_{\nu_{2}}$. By the induction hypothesis, we have $\delta_{\nu_{2}}=\delta_{\nu_{2}^{\prime}} \delta_{\nu_{2}^{\prime} \backslash \nu_{2}}$. As $\nu^{\prime} \backslash \nu=\left\{\nu_{1}\right\} \cup \nu_{2}^{\prime} \backslash \nu_{2}$, and as $\delta_{\nu_{2}^{\prime}}$ commutes to $\delta_{\nu_{1}}$ (since $\overline{\nu_{2}^{\prime}} \cap \overline{\nu_{1}}=\emptyset$ ), we get the result by induction.

(ii): Denote by $\nu$ the only nontrivial part of $\lambda^{\prime}$, and by $\lambda_{1}, \ldots, \lambda_{l}$ the nontrivial parts of $\lambda$. To prove the result, the essential step is to notice that since for $i>1$ we have $\overline{\lambda_{i}} \cap \overline{\lambda_{1}}=\emptyset$, each $\lambda_{i}$ lies inside a single connected component of the complement of $\overline{\lambda_{1}}$ in $\bar{\nu}$, thus each is included in a part of $\lambda_{1} \backslash \nu$. Thus (ii) follows by induction on the number of parts of $\lambda$ from (i). The uniqueness comes from the fact that the identity $\delta_{\lambda} \delta_{\lambda \backslash \lambda^{\prime}}=\delta_{\lambda^{\prime}}$ is valid in $M_{x} \subset B_{x}$, and $B_{x}$ is a group.

Finally, (iii) is easily obtained by applying (ii) to all the nontrivial parts of $\lambda^{\prime}$.

For $\sigma \in \mathfrak{S}_{x}$, we denote by $|\sigma|$ the minimum number of transpositions in a decomposition of $\sigma$ as a product of transpositions; such a decomposition is called reduced (note that we allow all transpositions). The map $\sigma \mapsto|\sigma|$ is not a morphism, but we have the relation $\left|\sigma_{1} \sigma_{2}\right| \leq\left|\sigma_{1}\right|+\left|\sigma_{2}\right|$.

\section{Lemma 1.12.}

(i) Let $\lambda \vdash x$ be the orbit decomposition of $\sigma \in \mathfrak{S}_{x}$. We have

$$
|\sigma|=\sum_{\nu \in \lambda}(|\nu|-1)=|x|-|\lambda|
$$


(ii) Let $\sigma \in \mathfrak{S}_{x}$. Let $t=\left(z, z^{\prime}\right)$. If $z$ and $z^{\prime}$ are in the same orbit of $\sigma$, then $|\sigma t|=|\sigma|-1$. If $z$ and $z^{\prime}$ are in different orbits of $\sigma$, then $|\sigma t|=|\sigma|+1$.

(iii) Let $\sigma \in \mathfrak{S}_{x}$. Let $t_{1} t_{2} \ldots t_{|\sigma|}$ be a reduced decomposition of $\sigma$. Then if $t_{i}=\left(z, z^{\prime}\right)$ is one of the transpositions, $z$ and $z^{\prime}$ belong to the same orbit of $\sigma$. Let $\lambda \vdash x$ be the orbit decomposition of $\sigma$ and, for $i=1, \ldots,|\sigma|$, $\lambda_{i} \vdash x$ be the orbit decomposition of $t_{1} t_{2} \ldots t_{i}$. Then $\lambda_{i} \prec \lambda$.

(iv) For all $m \in M_{x}$, we have $l(m) \geq\left|\phi_{x}(m)\right|$.

(v) If $\lambda=x$, we have $l\left(\delta_{\lambda}\right)=\left|\phi_{x}\left(\delta_{\lambda}\right)\right|$.

Proof. (i) is easy.

(ii) comes from (i) and the following remark: When $z$ and $z^{\prime}$ are in the same orbit, multiplying by $t$ splits this orbit into two orbits, thus increasing by 1 the number of orbits; when $z$ and $z^{\prime}$ are in different orbits, multiplying by $t$ merges their orbits, thus decreasing by 1 the number of orbits.

(iii) is an easy induction from (ii) and its proof: As the decomposition is reduced, the relation $|\sigma|=\left|t_{1} t_{2} \ldots t_{|\sigma|}\right|$ can only be achieved if the successive multiplications by the $t_{i}$ merge orbits.

(iv) and (v) are easy.

We can now prove Proposition 1.8:

Proof. (i) Let $\lambda, \lambda^{\prime} \in \mathcal{P}_{x}$. As the converse implication is trivial, we only have to check that

$$
\left(\exists m^{\prime \prime} \in M_{x}, \delta_{\lambda} m^{\prime \prime}=\delta_{\lambda^{\prime}}\right) \Rightarrow\left(\exists \lambda^{\prime \prime} \in \mathcal{P}_{x}, \delta_{\lambda} \delta_{\lambda^{\prime \prime}}=\delta_{\lambda^{\prime}}\right) .
$$

Let $m^{\prime \prime} \in M_{x}$ such that $\delta_{\lambda} m^{\prime \prime}=\delta_{\lambda^{\prime}}$. Consider the image in $\mathfrak{S}_{x}$ of this identity: $\phi_{x}\left(\delta_{\lambda}\right) \phi_{x}\left(m^{\prime \prime}\right)=\phi_{x}\left(\delta_{\lambda^{\prime}}\right)$. By Lemma $1.12(\mathrm{v})$, we have $\left|\phi_{x}\left(\delta_{\lambda}\right)\right|=l\left(\delta_{\lambda}\right)$ and $\left|\phi_{x}\left(\delta_{\lambda^{\prime}}\right)\right|=l\left(\delta_{\lambda^{\prime}}\right)$, and, using Lemma 1.12 (iv), we have $\left|\phi_{x}\left(\delta_{\lambda^{\prime}}\right)\right|=$ $l\left(\delta_{\lambda^{\prime}}\right)=l\left(\delta_{\lambda} m^{\prime \prime}\right)=l\left(\delta_{\lambda}\right)+l\left(m^{\prime \prime}\right) \geq\left|\phi_{x}\left(\delta_{\lambda}\right)\right|+\left|\phi_{x}\left(m^{\prime \prime}\right)\right|$ and thus $\left|\phi_{x}\left(\delta_{\lambda^{\prime}}\right)\right|=$ $\left|\phi_{x}\left(\delta_{\lambda}\right)\right|+\left|\phi_{x}\left(m^{\prime \prime}\right)\right|$. Consequently, when concatenating reduced decompositions for $\phi_{x}\left(\delta_{\lambda}\right)$ and $\phi_{x}\left(m^{\prime \prime}\right)$, one gets a reduced decomposition for $\phi_{x}\left(\delta_{\lambda^{\prime}}\right)$. By Lemma 1.12 (iii), this implies that the orbit decomposition for $\phi_{x}\left(\delta_{\lambda}\right)$ is finer than the one for $\phi_{x}\left(\delta_{\lambda^{\prime}}\right)$, i.e., $\lambda \prec \lambda^{\prime}$. We conclude by Lemma 1.11 (iii).

(ii) By Lemma 1.11 (iii), the map $D$ is a poset morphism. It is injective: From $\delta_{\lambda}$, one recovers $\lambda$ by considering the orbit decomposition of $\phi_{x}\left(\delta_{\lambda}\right)$. The fact that the inverse map is a poset morphism, i.e., $\delta_{\lambda} \prec \delta_{\lambda^{\prime}} \Rightarrow \lambda \prec \lambda^{\prime}$, has already been obtained in our proof of (i).

Remark 1.13. If $x$ is convex, then there is a largest element $\delta_{\{x\}}$ in the poset $\left(P_{x}, \prec\right.$ ) (which corresponds to the coarsest partition which has just one part equal to $x)$.

\section{Pre-Garside structures and Garside monoids.}

The existence of nice normal forms in the Birman-Ko-Lee monoid will be of crucial importance in the proof of our main theorem. This property is 
proved in [BiKoLee], and we could have just quoted and translated it into our setting. However, it appeared that our geometric interpretation allows us to give a new proof, far less computational, of some of the main results in [BiKoLee]. The Birman-Ko-Lee monoid is a Garside monoid, in the sense of $[\mathbf{D e P a}]$, and this implies (among others) the existence of the normal form. To give a simple proof of this fact, we make use a new criterion of "Garsiditude". This criterion relies on the notion of pre-Garside structure, which can be seen as an axiomatization of the context in which most of the proofs in $[\mathrm{Mi}]$ are actually valid.

When $A$ and $B$ are two sets, we mean by "partial map" from $A$ to $B$ a datum consisting of a subset $A^{\prime} \subset A$ and a map $f: A^{\prime} \rightarrow B$. It is convenient to refer to $A^{\prime}$ implicitly, and to use a slightly abusive language, e.g., we will write " $f(a)$ is defined" instead of " $a \in A^{\prime \prime}$ ".

Definition 2.1. Let $P$ be a set. An atomic partial product on $P$ is a partial map $m: P \times P \rightarrow P$ (we will denote $m(a, b)$ by $a . b$ or $a b$ ), satisfying the following axioms:

(i) (Unit element and associativity.) There exists an element $1 \in P$ such that for all $a \in P$, both 1. $a$ and $a .1$ exist and are equal to $a$. For any $a, b, c \in P$, it is equivalent for $a b$ and $(a b) c$ to be defined or for $b c$ and $a(b c)$ to be defined and then $a(b c)=(a b) c$.

(ii) (Finite number of atoms.) Let $P^{*}=P-\{1\}$; the image of $P^{*} \times P^{*}$ is in $P^{*}$, and the complement $S=P^{*}-m\left(P^{*} \times P^{*}\right)$ is finite (the elements of $S$ are the atoms of $(P, m))$.

(iii) (Grading.) There exists a function $l: P \rightarrow \mathbf{N}$ such that $p \in P^{*} \Rightarrow$ $l(p)>0$ and $l(a b) \geq l(a)+l(b)$ whenever $a b$ is defined.

If $a, b \in P$ are such that $a b$ is defined, we say that $a$ (resp. $b$ ) is a left (resp. right) divisor of $a b$. Conversely, $a b$ is a right (resp. left) multiple of $a$ (resp. b).

Definition 2.2. Suppose $P$ is a set together with an atomic partial product. The associated monoid $M(P)$ is the monoid defined by the following presentation:

- As a set of generators we take $P$.

- For relations we take $a b=c$ whenever $a, b, c \in P$ are such that $a b$ is defined in $P$ and equal to $c$.

Note that if $P$ is a subset of a monoid $M^{\prime}$, and if the partial product on $P$ is a restriction of the monoid law in $M^{\prime}$, then there is a natural morphism $M(P) \rightarrow M^{\prime}$. If $P$ generates $M^{\prime}$, the morphism is surjective.

As in [Mi], we note that we can identify $M(P)$ to the set of finite sequences of elements of $P$, quotiented by the minimal equivalence relation $\sim$ such that

$$
\left(p_{1}, \ldots, p_{i-1}, p_{i}, p_{i+1}, \ldots, p_{n}\right) \sim\left(p_{1}, \ldots, p_{i-1}, a, b, p_{i+1}, \ldots, p_{n}\right)
$$


whenever the product $a b$ is defined and equal to $p_{i}$.

Let $\left(x_{1}, \ldots, x_{n}\right)$ be a sequence of elements of $P$. There are $(2(n-1)) ! /(n !(n$ $-1)$ !) different ways to put brackets on the product $x_{1} \ldots x_{n}$. By an obvious induction from the associativity axiom, if the product is defined in $P$ for one of these bracketings, then it is defined in $P$ for any other bracketing, and the value of this product does not depend on the choice of the bracketing. When this is the case, we write $x_{1} \ldots x_{n}$ for this product.

Lemma 2.3. Let $\left(x_{1}, \ldots, x_{n}\right)$ be a sequence of elements of $P$ equivalent, in $M(P)$, to a single term sequence $(y)$. Then the product $x_{1} \ldots x_{n}$ is defined in $P$ and we have $x_{1} \ldots x_{n}=y$.

Proof. By assumption $\left(x_{1}, \ldots, x_{n}\right)$ can be transformed into $(y)$ by a finite rewriting process

$$
l_{0}=\left(x_{1}, \ldots, x_{n}\right) \rightarrow l_{1} \rightarrow \cdots \rightarrow l_{k}=(y)
$$

in which, at each step, the elementary transformation $l_{j-1} \rightarrow l_{j}$ is

- either of the type

$$
\left(p_{1}, \ldots, p_{i-1}, a, b, p_{i+1}, \ldots, p_{m}\right) \rightarrow\left(p_{1}, \ldots, p_{i-1}, p_{i}, p_{i+1}, \ldots, p_{m}\right)
$$

- or of the type

$$
\left(p_{1}, \ldots, p_{i-1}, p_{i}, p_{i+1}, \ldots, p_{m}\right) \rightarrow\left(p_{1}, \ldots, p_{i-1}, a, b, p_{i+1}, \ldots, p_{m}\right)
$$

where $a, b$ are such that their product is defined in $P$ and equal to $p_{i}$. Suppose the length $k$ of the rewriting process is minimal.

Suppose some of the transformations are of the second type, and chose $j$ maximal such that $l_{j-1} \rightarrow l_{j}$ is of the second type

$$
\left(p_{1}, \ldots, p_{i-1}, p_{i}, p_{i+1}, \ldots, p_{m}\right) \rightarrow\left(p_{1}, \ldots, p_{i-1}, a, b, p_{i+1}, \ldots, p_{m}\right) .
$$

As all further steps are of the first type, we have $k=j+m$ and the product $p_{1} \ldots p_{i-1} a b p_{i+1} \ldots p_{m}$ is defined in $P$ (and is equal to $y$ ). Choosing a bracketing starting by $\ldots(a b) \ldots$, we see that the product $p_{1} \ldots p_{i-1} p_{i} p_{i+1} \ldots p_{m}$ must also be defined in $P$ (and equal to $y$ ). But this yields a rewriting of length $j+m-2<k$ and we have a contradiction.

Thus there are no transformation of the second type in a minimal rewriting. The result follows.

The following proposition is a straightforward consequence of the lemma:

\section{Proposition 2.4.}

(i) The natural map from $P$ to $M(P)$ is injective.

(ii) If $x \in M(P)$ divides $a \in P$ then $x \in P$.

A right common multiple of $s$ and $t$ is an element $u$ which is a right multiple of both $s$ and $t$. A common right multiple $u$ of $s$ and $t$ is a least right common multiple if and only if, for all common right multiple $v$ of $s$ 
and $t, u$ is a left divisor of $v$. Similarly, there are notions of (least) left common multiple, of (greatest) right common divisor and of (greatest) left common divisor.

Definition 2.5. A pre-Garside structure on a set $P$ is an atomic partial product, with set of atoms $S$, satisfying the following additional axioms:

(iv) If two elements of $S$ have a common right multiple in $P$, they have a least common right multiple. When $s, t \in S$ have a least right common multiple, we write it $\Delta_{s, t}$.

$\left(\mathrm{iv}^{\prime}\right)$ If two elements of $S$ have a common left multiple in $P$, they have a least common left multiple.

(v) If $s, t \in S$ have a common right multiple in $P$, and if $a \in P$ is such that $a s \in P$ and $a t \in P$, then $a \Delta_{s, t} \in P$.

(vi) For all $m \in M(P)$ and $a, b \in P$, if either $a m=b m$ or $m a=m b$, then $a=b$.

In [Mi], $S$ was taken to be the set of usual Artin generators, and $P$ was the set of reduced braids. To handle the Birman-Ko-Lee monoid, we will take for $S$ the set $S_{\mu_{n}}$ and for $P$ the set $P_{\mu_{n}}$.

Condition (v) is satisfied e.g., if there exists a common right multiple in $P$ of all elements of $P$, which is the case in the usual braid monoid and also in the Birman-Ko-Lee monoid (see 1.13). Note that such an element is necessarily unique and if it exists, the finiteness of $S$ implies that $P$ is finite. We shall see (2.22) that its existence is equivalent to the fact that all pairs of elements of $S$ have a right lcm.

The following lemmas and propositions are rephrasings of [Mi], 1.4 to 1.9.

Lemma 2.6. Let $X$ be a finite subset of $M(P)$ such that:

- If $x \in X, a \in M(P), a \prec x$ then $a \in X$.

- If $a \in M(P), s, t \in S$, as, at $\in X$, then $\Delta_{s, t}$ exists and $a \Delta_{s, t} \in X$.

Then there exists $g \in X$ such that $X$ is the set of left divisors of $g$.

Proof. The statement follows $[\mathrm{Mi}] 1.4$, and the proof is exactly the same (after having extended the length function to $M(P)$ by setting $l(m):=$ $\left.\max \left\{\sum_{i=1}^{n} l\left(p_{i}\right) \mid p_{1}, \ldots, p_{n} \in P, m=p_{1} \ldots p_{n}\right\}\right)$.

Proposition 2.7. Any two elements of $P$ have a left g.c.d. in P.

Proof. We can follow the proof of [Mi] 1.6, replacing 1.5 in [Mi] by Axiom (iv).

We shall denote by $a \wedge b$ the left g.c.d. of $a$ and $b$.

Proposition 2.8. For $a$ and $b$ in $P$ there exists a unique maximal $c \prec b$ such that $a c \in P$. 
Proof. We apply Lemma 2.6 to the set $X$ of $c$ such that $c \prec b$ and $a c \in P$. To check the assumptions of that lemma, we need that if $s, t \in S$ and if $c s, c t \in X$ then $\Delta_{s, t}$ exists and $a c \Delta_{s, t} \in P$ (then we have $c \Delta_{s, t} \in X$ ). Since $c s$ and ct divide $b \in P$, by cancellability and $2.4 s$ and $t$ have a common multiple in $P$, so by Axiom (iv) $\Delta_{s, t}$ exists and by Axiom (v) $a c \Delta_{s, t} \in P$.

Definition 2.9. In the situation of 2.8 we denote by $\alpha_{2}(a, b)$ the element $a c$ and we denote by $\omega_{2}(a, b)$ the unique $d \in P$ such that $b=c d$. We thus have $a b=\alpha_{2}(a, b) \omega_{2}(a, b)$.

Note that the uniqueness of $\omega_{2}$ follows from Axiom (vi).

Proposition 2.10. For $a, b, c, a b \in P$ we have $\alpha_{2}(a b, c)=\alpha_{2}\left(a, \alpha_{2}(b, c)\right)$.

Proof. The statement is [Mi], 1.8 and the proof is the same.

Proposition 2.11. For $a, b, c, a b \in P$ we have

$$
\omega_{2}(a b, c)=\omega_{2}\left(a, \alpha_{2}(b, c)\right) \omega_{2}(b, c) .
$$

Proof. By Propositions 2.8 and 2.10 the products of both sides with $\alpha_{2}(a b, c)$ are equal. By Axiom (vi) we will be done if we show that $\omega_{2}\left(a, \alpha_{2}(b, c)\right) \omega_{2}(b, c)$ is in $P$. By definition 2.9 there exists $z \in P$ such that $\alpha_{2}(b, c)=b z$ and $c=z \omega_{2}(b, c)$. As $a b \prec a b z$ and $a b \in P$ we have $\alpha_{2}(a, b z)=a b z_{1}$ for some $z_{1} \prec z$ (by definition of $\alpha_{2}$ and cancellability in $P$ ). Hence $b z=b z_{1} \omega_{2}(a, b z)$ and $z=z_{1} \omega_{2}(a, b z)$. So $z_{1} \omega_{2}\left(a, \alpha_{2}(b, c)\right) \omega_{2}(b, c)=z_{1} \omega_{2}(a, b z) \omega_{2}(b, c)=$ $z \omega_{2}(b, c)=c$. The result follows since any divisor of $c$ is in $P$ by 2.4 .

We now extend the definition of $\alpha_{2}$ to $M(P)$, following [Mi], 2.1 to 2.6. All the proofs of [Mi] can be reproduced, replacing Proposition 1.5 in [Mi] by Axiom (iv).

Proposition 2.12. There is a unique function $\alpha: M(P) \rightarrow P$ extending the identity of $P$ and satisfying $\alpha(a b)=\alpha_{2}(a, b)$ for $a, b \in P$ and $\alpha(g h)=$ $\alpha(g \alpha(h))$ for $g, h \in M(P)$. Moreover $\alpha(g)$ is the unique maximal element in $\{c \in P \mid c \prec g\}$.

Proposition 2.13. There exists a unique function $\omega: M(P) \rightarrow M(P)$ such that $\omega(a b)=\omega(a, b)$ for $a, b \in P($ in particular $\omega(a)=1$ for $a \in P)$ and such that $\omega(g h)=\omega(g \alpha(h)) \omega(h)$ for $g, h \in M(P)$.

Proposition 2.14. Let $g \in M(P)$; then $\omega(g)$ is the unique $y \in M(P)$ such that $g=\alpha(g) y$.

Proposition 2.15. The monoid $M(P)$ has left and right cancellation property (i.e., in Axiom (vi) we can replace the condition $a, b \in P$ by $a, b \in$ $M(P))$.

Proposition 2.16. If $s, t \in S$ divide $a \in M(P)$ on the left, then $\Delta_{s, t}$ exists and divides a. 
Proposition 2.17. For $a, b \in M(P)$ there exists a unique maximal $c \in$ $M(P)($ for $\prec)$ such that $c \prec a$ and $c \prec b$.

We shall still denote this left g.c.d. by $a \wedge b$.

Proposition 2.18. A family of elements of $M(P)$ which has a right (resp. left) common multiple has a right (resp. left) lcm in $M(P)$.

Proof. Assume that all elements of the family $\left\{a_{i}\right\}_{i \in I}$ divide $c$. If we can apply 2.6 to the set $X$ of elements of $M(P)$ which divide all common multiples of the $a_{i}$, it will give the result. Let us check the assumption of 2.6. This set $X$ is finite as it is included in the set of divisors of $c$. The first assumption of 2.6 is clearly satisfied. The second assumption is a consequence of the fact that if $s, t \in S$ are such that $x s$ and $x t$ divide some element $x z$, then $s$ and $t$ divide $\alpha(z)$, so $\Delta_{s, t}$ exists and divides $z$, whence $x \Delta_{s, t}$ divides $x z$.

We have a more precise result for elements of $P$ :

Proposition 2.19. If a family of elements of $P$ has a common right (resp. left) multiple in $M(P)$ then its right (resp. left) lcm exists and is in $P$.

Proof. The lcm exists by the preceding proposition. Let $m$ be this lcm. Any divisor of $m$ divides $\alpha(m) \in P$, whence the result.

We now get a normal form for any element of $M(P)$ exactly as in [Mi].

Definition 2.20. A decomposition $\left(g_{1}, \ldots, g_{n}\right)$ of an element $g_{1} \ldots g_{n}$ of $M(P)$ is said to be its normal form if no $g_{i}$ is equal to 1 and for any $i$ we have $g_{i}=\alpha\left(g_{i} \ldots g_{n}\right)$.

The following statement is 4.2 of $[\mathbf{M i}]$ and the same proof applies.

Proposition 2.21. A decomposition $\left(g_{1}, \ldots, g_{i}\right)$ with $g_{i} \in P$ is a normal form if and only if $\left(g_{i}, g_{i+1}\right)$ is a normal form for any $i$. In particular any segment $\left(g_{i}, \ldots, g_{j}\right)$ of a normal form $\left(g_{1}, \ldots, g_{n}\right)$ is a normal form.

In the same way, statements 4.6 to 4.9 and 5.1 to 5.3 of [Mi] generalize to our setting.

Assume that all elements of $P$ have a right common multiple (which is in $P$ by 2.19). By 2.18, this is the same as assuming that any finite subset of elements of $P$ has a $\mathrm{lcm}$. As already noticed, the existence of a $\mathrm{lcm} \Delta$ of all elements in $P$ implies the finiteness of $P$. The converse is not true, but we have:

Proposition 2.22. The elements of $P$ have a common right multiple if and only if $P$ is finite and any pair of elements of $S$ has a common multiple.

Proof. Implication "only if" is clear. The converse is an immediate application of 2.6, taking $X=P$. 
By 2.12, for any $a \in M(P)$ we have $\alpha(a)=a \wedge \Delta$.

Proposition 2.23. Assume $P$ has a right lcm $\Delta$.

- There is an automorphism $x \mapsto \bar{x}$ of $M(P)$ mapping $S$ to itself and such that $x \Delta=\Delta \bar{x}$.

- The element $\Delta$ is the left lcm of $P$.

Proof. It is sufficient to define the automorphism on $P$. As $\Delta$ is a right multiple of all elements of $P$, for any $a \in P$ there exists a unique $\Delta_{a} \in P$ such that $\Delta=a \Delta_{a}$ and there exists a unique $\bar{a} \in P$ such that $\Delta=\Delta_{a} \bar{a}$, so that $a \Delta=a \Delta_{a} \bar{a}=\Delta \bar{a}$. The map $a \mapsto \bar{a}$ is injective by cancellability in $M(P)$ and is thus compatible with the product. As $P$ is finite, it is surjective. If $a \in S$, by surjectivity $\bar{a}$ cannot be the product of two nontrivial elements of $P$, so has to be in $S$ by Axiom (ii).

As $a \mapsto \bar{a}$ is a bijection of $P$, the above proof shows that $\Delta$ is a left multiple of all elements of $P$. So it is the left lcm of $P$.

We can now compare our formalism with the one in [DePa]: If $P$ is a preGarside structure with right common multiples, then, it is readily seen that any two elements of $M(P)$ have left and right common multiples: Indeed, if $m_{1}, m_{2} \in M(P)$ are both products of $n$ or less elements of $S$, then $\Delta^{n}$ is a left and right multiple of both $m_{1}$ and $m_{2}$ (use Proposition 2.22: The conjugation by $\Delta$ maps $S$ into itself). Thus, by Proposition 2.18, pairs of elements in $M(P)$ have both a right lcm and a left lcm. Moreover, by Proposition 2.22, $\Delta$ is both the right $\mathrm{lcm}$ and the left $\mathrm{lcm}$ of $P$. Conversely, elements of $P$ are left and right divisors of $\Delta$, and, by Proposition 2.4, $\Delta$ has no other left or right divisors. We have proved that $M(P)$ is a Garside monoid, as defined in [DePa].

Conversely, if $M$ is a Garside monoid, then the restriction of the monoid product to the set $P$ of simple elements (see $[\mathbf{D e P a}]$ ) is a pre-Garside structure with common multiples.

We have proved the:

Theorem 2.24. Let $P$ be set, endowed with a pre-Garside structure, such that all atoms have a common right multiple. Then $M(P)$ is a Garside monoid.

Conversely, when $M$ is a Garside monoid with fundamental element $\Delta$, the set $P$ of divisors of $\Delta$ has a pre-Garside structure for the partial product obtained by restriction of the product in $M$; all elements of $P$ have a common right multiple and $M \simeq M(P)$.

Note that our approach already gives interesting results (e.g., 2.26 below) even when some elements of $P$ have no common multiple, e.g., in the case of braid groups attached to infinite type Coxeter groups; in this case, $M(P)$ is no longer a Garside monoid. More important is, for the present article, 
that pre-Garside structures provide us with a convenient criterion to check that the Birman-Ko-Lee monoid is a Garside monoid.

Let us recall the following proposition about Garside monoids, which is already in $[\mathbf{D e P a}]$.

Proposition 2.25. Assume that $P$ has a right lcm. The monoid $M(P)$ injects into the group $G(P)$ having the same presentation and any element of $G$ can be written uniquely as $x^{-1} y$ with $x, y \in M(P)$ and $x \wedge y=1$.

Proof. The statement follows [Mi] 3.2 and the same proof applies.

We now generalize $[\mathrm{Mi}]$ 4.4.

Proposition 2.26. Let $\Gamma$ be a group of automorphisms of $M(P)$ stabilizing $S$. Let $\Sigma$ be the set of lcms of $\Gamma$-orbits in $S$ which exist and are not the product of other such lcms; then $P^{\Gamma}$ has a pre-Garside structure with atoms $\Sigma$; the monoid $M(P)^{\Gamma}$ identifies with $M\left(P^{\Gamma}\right)$. If moreover we assume that $P$ has a right lcm then the group $G(P)^{\Gamma}$ identifies with $G\left(P^{\Gamma}\right)$ (cf. 2.25).

Proof. Axiom (i) holds as it holds in $P$ and as the product of two $\Gamma$-fixed elements is $\Gamma$-fixed. Let $x \in P^{\Gamma}$, and let $s \in S$ such that $s \prec x$; then $x$ is divisible by all elements in the orbit of $s$, so is divisible by their lcm (which exists). So $P^{\Gamma}$ is generated by the lcms of $\Gamma$-orbits in $S$ which exist, thus by $\Sigma$, and we have arranged for elements of $\Sigma$ to be atoms, so Axiom (ii) is satisfied. The length inherited from $P$ is still compatible with the product so we have Axiom (iii) (but note that the elements of $\Sigma$ may have length greater than 1 even if all elements of $S$ had length 1). The lcm of two elements of $P^{\Gamma}$ is $\Gamma$-fixed by its uniqueness and is in $P$ by 2.19 , whence Axioms (iv) and (iv'). If $\sigma$ and $\tau$ are in $\Sigma$ and have a lcm $\Delta_{\sigma, \tau}$ and if $a \in P^{\Gamma}$ is such that $a \sigma$ and $a \tau$ are in $P$ then $a \Delta_{\sigma, \tau}$ is the lcm of $a \sigma$ and $a \tau$ so is in $P$ by 2.19 , and is clearly $\Gamma$-fixed, whence Axiom (v). The monoid $M\left(P^{\Gamma}\right)$ is by definition a submonoid of $M(P)$, so Axiom (vi) holds.

Let $x \in M(P)^{\Gamma}$; the uniqueness of its normal form implies that each term is in $P^{\Gamma}$. On the other hand, if $x \in M(P)^{\Gamma}$, as $\alpha(x)$ is the unique maximal element in $P$ dividing $x$ it is also the unique maximal element in $P^{\Gamma}$ dividing $x$, so, by the definition of normal forms, the normal form of an element of $M(P)^{\Gamma}$ is a normal form in $M\left(P^{\Gamma}\right)$. This shows that $M(P)^{\Gamma}$ identifies with $M\left(P^{\Gamma}\right)$. The same argument shows that $G(P)^{\Gamma}$ identifies with $G\left(P^{\Gamma}\right)$ when $\Delta$ exists, as $\Delta$ is in $P^{\Gamma}$.

\section{The Birman-Ko-Lee monoid.}

Let $x \in X_{n}$, and let $S_{x}, P_{x}$ and $M_{x}$ as defined as in Section 1 .

Lemma 3.1. The restriction of the product in $M_{x}$ defines an atomic partial product in $P_{x}$ with set of atoms $S_{x}$. 
Proof. Axiom (i) is a consequence of the associativity in the monoid $M_{x}$ and of 1.8(i). Axioms (ii) and (iii) are easy consequences of the existence of the length function $l: M_{x} \rightarrow \mathbf{N}$ introduced in Section 1. The atoms are clearly, by construction, the elements of $S_{x}$.

We call the monoid $M_{\mu_{n}}$ the Birman-Ko-Lee monoid (whenever $x$ is convex, we have $M_{x} \simeq M_{\mu_{n}}$ ).

Remark 3.2. If we map the base point $\mu_{n}$ to the usual base point $\{1, \ldots, n\}$ by choosing $z_{1} \in \mu_{n}$ and numbering clockwise the elements of $\mu_{n}$ starting at $z_{1}$ as $z_{1}, z_{2}, \ldots, z_{n}$, then the $\delta_{\left\{z_{i}, z_{i+1}\right\}}$ map to the usual Artin generators $\sigma_{i}$ of $\pi_{1}\left(X_{n}, \mu_{n}\right)$ and the $\delta_{\left\{z_{i}, z_{j}\right\}}$ map to the generators $a_{i j}$ considered in [BiKoLee] (note that they always suppose $i>j$ while we don't order the pairs $\left.\left\{z, z^{\prime}\right\}\right)$. Then the elements of $P_{\mu_{n}}$ map to the canonical factors defined in [BiKoLee]. In order to compare our definition to that of [BiKoLee] one should note that [BiKoLee] call $a_{i j}, a_{k l}$ an "obstructing pair" exactly when the partition $\left\{\left\{z_{i}, z_{j}\right\},\left\{z_{k}, z_{l}\right\}\right\}$ is crossing.

The monoid $M_{\mu_{n}}$ enjoys remarkable properties which are consequences of the discussion in the previous section and of the following theorem.

Theorem 3.3. The restriction of the product in $M_{\mu_{n}}$ to a partial product in $P_{\mu_{n}}$ is a pre-Garside structure, and $P_{\mu_{n}}$ has a lcm $\delta$.

Proof. Since $\mu_{n}$ is convex, $\delta=\delta_{\left\{\mu_{n}\right\}}$ is an lcm of $P_{\mu_{n}}$, as explained in 1.13. This eliminates the need to check Axiom (v).

Let us now prove Axiom (iv). Using the isomorphism in Proposition 1.8, and noticing that any pair of elements of $\mu_{n}$ defines a non-crossing edge, we have to prove: For all $z_{1}, z_{1}^{\prime}, z_{2}, z_{2}^{\prime} \in \mu_{n}$, the set

$$
E=\left\{\lambda \models \mu_{n} \mid\left\{\left\{z_{1}, z_{1}^{\prime}\right\}\right\} \prec \lambda \text { and }\left\{\left\{z_{2}, z_{2}^{\prime}\right\}\right\} \prec \lambda\right\}
$$

has a minimum element for $\prec$. We discuss by cases:

- Assume $\left[z_{1}, z_{1}^{\prime}\right] \cap\left[z_{2}, z_{2}^{\prime}\right]=\emptyset$. Then let $\lambda=\left\{\left\{z_{1}, z_{1}^{\prime}\right\},\left\{z_{2}, z_{2}^{\prime}\right\}\right\}$. Clearly, $\lambda \in E$, and any partition coarser than $\left\{\left\{z_{1}, z_{1}^{\prime}\right\}\right\}$ and $\left\{\left\{z_{2}, z_{2}^{\prime}\right\}\right\}$ must be coarser than $\lambda$.

- Assume $\left[z_{1}, z_{1}^{\prime}\right] \cap\left[z_{2}, z_{2}^{\prime}\right] \neq \emptyset$. Let $\lambda=\left\{\left\{z_{1}, z_{1}^{\prime}, z_{2}, z_{2}^{\prime}\right\}\right\}$ (the nontrivial part may have three or four elements). Clearly $\lambda \in E$. Now let $\lambda^{\prime} \in E$. Consider $\nu_{1}$ the part of $\lambda^{\prime}$ in which $z_{1}$ lies, $\nu_{2}$ the part of $\lambda^{\prime}$ in which $z_{2}$ lies. Because $\left\{\left\{z_{1}, z_{1}^{\prime}\right\}\right\} \prec \lambda^{\prime}$ and $\left\{\left\{z_{2}, z_{2}^{\prime}\right\}\right\} \prec \lambda^{\prime}$, we have $z_{1}^{\prime} \in \nu_{1}$ and $z_{2}^{\prime} \in \nu_{2}$. As $\lambda^{\prime}$ is non-crossing, we must have $\nu_{1}=\nu_{2}$ (otherwise we would have $\overline{\nu_{1}} \cap \overline{\nu_{2}}=\emptyset$, which contradicts $\left.\left[z_{1}, z_{1}^{\prime}\right] \cap\left[z_{2}, z_{2}^{\prime}\right] \neq \emptyset\right)$. Thus $\lambda$ is finer than $\lambda^{\prime}$.

$\left(\mathrm{iv}^{\prime}\right)$ is proved similarly.

To prove (vi), consider the natural morphism $M\left(P_{\mu_{n}}\right) \rightarrow M_{\mu_{n}}$ and its composition with the epimorphism $M_{\mu_{n}} \rightarrow \mathfrak{S}_{\mu_{n}}$. Let $m \in M\left(P_{\mu_{n}}\right), \lambda, \lambda^{\prime} \models$ 
$\mu_{n}$ such that either $\delta_{\lambda} m=\delta_{\lambda^{\prime}} m$ or $m \delta_{\lambda}=m \delta_{\lambda^{\prime}}$. Denote by $\sigma$ the image of $m$ in $\mathfrak{S}_{\mu_{n}}$. We have $\phi_{\mu_{n}}\left(\delta_{\lambda}\right) \sigma=\phi_{\mu_{n}}\left(\delta_{\lambda^{\prime}}\right) \sigma$ or $\sigma \phi_{\mu_{n}}\left(\delta_{\lambda}\right)=\sigma \phi_{\mu_{n}}\left(\delta_{\lambda^{\prime}}\right)$. As $\mathfrak{S}_{\mu_{n}}$ is a group, this implies $\phi_{\mu_{n}}\left(\delta_{\lambda}\right)=\phi_{\mu_{n}}\left(\delta_{\lambda^{\prime}}\right)$. By Lemma 1.10, the restriction of $\phi_{\mu_{n}}$ to $P_{\mu_{n}}$ is injective, thus we have as required $\delta_{\lambda}=\delta_{\lambda^{\prime}}$.

Remark 3.4. According to the isomorphism in Proposition 1.8, the above theorem implies that the poset of non-crossing partitions of $\mu_{n}$ is a lattice. If $\lambda_{1}, \lambda_{2} \models \mu_{n}$, the set

$$
E=\left\{\lambda\left|=\mu_{n}\right| \lambda_{1} \prec \lambda \text { and } \lambda_{2} \prec \lambda\right\}
$$

admits a minimum element. We leave to the reader to check that this can be proved directly, using the following arguments: Given two (possibly crossing) partitions, the set of (possibly crossing) partitions coarser than the two partitions admits a minimum element; given a (possibly crossing) partition, the set of non-crossing partitions which are coarser admits a minimum element.

Of course, by construction, $M_{x}$ embeds in $B_{x}$ for all $x \in X_{n}$. What is specific to the Birman-Ko-Lee monoid is the following result, which is the analog in our intrinsic setting of the embedding theorem in [BiKoLee].

Corollary 3.5. The natural morphism $M\left(P_{\mu_{n}}\right) \rightarrow M_{\mu_{n}}$ is an isomorphism.

Proof. Consider the group $G\left(P_{\mu_{n}}\right)$ given by the group presentation corresponding to the monoid presentation of $M\left(P_{\mu_{n}}\right)$. As the partial product in $P_{\mu_{n}}$ is a restriction of the one in $B_{\mu_{n}}$, there is a natural morphism

$$
G\left(P_{\mu_{n}}\right) \rightarrow B_{\mu_{n}} \text {. }
$$

By Proposition 1.3, this morphism is surjective.

Note that it is easy to adapt Sergiescu's presentations to find a presentation of $B_{\mu_{n}}$ where the generators corresponds to the elements of $S_{\mu_{n}}$. A set of defining relations is for example given in [BiKoLee], Proposition 2.1:

- $\delta_{\left\{z_{1}, z_{1}^{\prime}\right\}} \delta_{\left\{z_{2}, z_{2}^{\prime}\right\}}=\delta_{\left\{z_{2}, z_{2}^{\prime}\right\}} \delta_{\left\{z_{1}, z_{1}^{\prime}\right\}}$ when $\left\{\left\{z_{1}, z_{1}^{\prime}\right\},\left\{z_{2}, z_{2}^{\prime}\right\}\right\}$ is non-obstructing,

- $\delta_{\left\{z_{1}, z_{2}\right\}} \delta_{\left\{z_{2}, z_{3}\right\}}=\delta_{\left\{z_{2}, z_{3}\right\}} \delta_{\left\{z_{3}, z_{1}\right\}}=\delta_{\left\{z_{3}, z_{1}\right\}} \delta_{\left\{z_{1}, z_{2}\right\}}$ for $z_{1}, z_{2}, z_{3}$ coming in clockwise order.

These relations are valid in $P_{\mu_{n}}$, thus in $G\left(P_{\mu_{n}}\right)$, and the morphism $G\left(P_{\mu_{n}}\right) \rightarrow$ $B_{\mu_{n}}$ is an isomorphism.

We conclude using the natural commutative diagram:

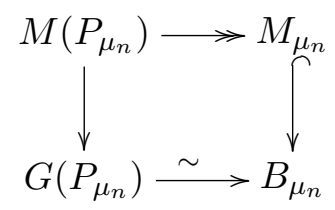

and the injectivity of the map $M\left(P_{\mu_{n}}\right) \rightarrow G\left(P_{\mu_{n}}\right)$ (Proposition 2.25). 
We identify $M\left(P_{\mu_{n}}\right)$ and $M_{\mu_{n}}$ through the natural isomorphism. As $M_{\mu_{n}}$ is a Garside monoid, we have nice normal forms, an algorithm for the word problem, ... The following property is the one we need for the proof of our main theorem.

Proposition 3.6. Let $\zeta=e^{\frac{2 i \pi}{n}}$.

(i) For $\lambda=\mu_{n}$ the automorphism of $M_{\mu_{n}}$ induced by $\delta$ maps $\delta_{\lambda}$ to $\delta_{\zeta \lambda}$.

(ii) For $d$ dividing $n$, the centralizer of $\delta^{n / d}$ in $\pi_{1}\left(X_{n}, \mu_{n}\right)$ is generated by the elements $\delta_{\lambda}$ for $\lambda \models \mu_{n}$ such that $\zeta^{n / d} \lambda=\lambda$.

Proof. It is an immediate consequence of 1.5 that (i) holds for a generator of the form $\delta_{\left\{s_{i}, s_{i+1}\right\}}$ where $s_{i}$ and $s_{i+1}$ are two consecutive points in a numbering of $\mu_{n}$. It follows that it holds for any generator $\delta_{\left\{s_{1}, s_{a}\right\}}$ by using that $\delta_{\left\{s_{1}, s_{a}\right\}}=\delta_{\left\{s_{1}, s_{2}\right\}} \ldots \delta_{\left\{s_{a-1}, s_{a}\right\}} \delta_{\left\{s_{a-2}, s_{a-1}\right\}}^{-1} \ldots \delta_{\left\{s_{1}, s_{2}\right\}}^{-1}$ (which follows also from 1.5), and it follows thus for any element of $P$. Part (ii) is then a consequence of 2.26 .

Remark. Note that 2.26 gives a "Birman-Ko-Lee" presentation of $C_{\mathbf{B}}\left(\delta^{i}\right)$. Let us work out an example to show that one has to take only a part of the lcms of $\delta^{i}$-orbits on $S$ to get an atomic set. Take $n=6$ and $i=2$. Then the $\delta^{2}$-orbits in $S$ are

$$
\begin{aligned}
&\left\{\delta_{\left\{s_{1}, s_{2}\right\}}, \delta_{\left\{s_{3}, s_{4}\right\}}, \delta_{\left\{s_{5}, s_{6}\right\}}\right\},\left\{\delta_{\left\{s_{2}, s_{3}\right\}}, \delta_{\left\{s_{4}, s_{5}\right\}}, \delta_{\left\{s_{1}, s_{6}\right\}}\right\}, \\
&\left\{\delta_{\left\{s_{1}, s_{3}\right\}}, \delta_{\left\{s_{3}, s_{5}\right\}}, \delta_{\left\{s_{1}, s_{5}\right\}}\right\} \text { and }\left\{\delta_{\left\{s_{1}, s_{4}\right\}}, \delta_{\left\{s_{2}, s_{5}\right\}}, \delta_{\left\{s_{3}, s_{6}\right\}}\right\}
\end{aligned}
$$

whose respective lcm are

$$
\delta_{\left(\left\{s_{1}, s_{2}\right\},\left\{s_{3}, s_{4}\right\},\left\{s_{5}, s_{6}\right\}\right)}, \delta_{\left(\left\{s_{2}, s_{3}\right\},\left\{s_{4}, s_{5}\right\},\left\{s_{1}, s_{6}\right\}\right)}, \delta_{\left\{s_{1}, s_{3}, s_{5}\right\}} \text { and } \delta ;
$$

but $\delta=\delta_{\left\{s_{1}, s_{3}, s_{5}\right\}} \delta_{\left(\left\{s_{1}, s_{2}\right\},\left\{s_{3}, s_{4}\right\},\left\{s_{5}, s_{6}\right\}\right)}$, so must be eliminated.

\section{A geometric normal form for canonical factors.}

Let $n$ be a positive integer. If $K_{1}, K_{2}$ are non-intersecting compact subsets of $\mathbf{C}$, we denote by $d\left(K_{1}, K_{2}\right)$ the positive number

$$
\inf _{\left(z_{1}, z_{2}\right) \in K_{1} \times K_{2}}\left|z_{1}-z_{2}\right| .
$$

It is clear that

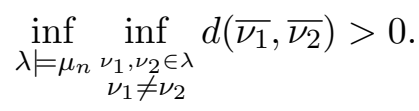

We define

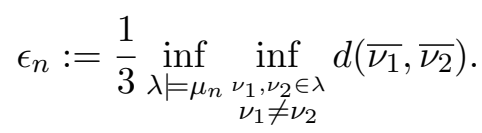

The exact value is not important, we will only use the fact that $\epsilon_{n}$ is fixed and small enough. In particular, the reader should check for himself that in the 
following definition, the strings do not intersect and thus $\gamma_{\lambda}$ is well-defined (note that the function $f$ has been defined in the first section).

Definition 4.1. Let $\lambda \models \mu_{n}$.

- Let $z \in \mu_{n}$. We define a path $\gamma_{z}:[0,1] \rightarrow \mathbf{C}$ in the following way:

- If $z$ is in a part of $\lambda$ with exactly one element then we set

$$
\forall t, \gamma_{z}(t)=z \text {. }
$$

- If $z$ is in a part of $\lambda$ with exactly two elements $z$ and $z^{\prime}$ we set:

$$
\forall t, \gamma_{z}(t)=f\left(z, z^{\prime}, \epsilon_{n}\right)(t)
$$

- If $z$ is in a part $\nu$ of $\lambda$ with three or more elements, we denote by $z^{\prime}$ the element of $\nu$ immediately after $z$ in the direct (i.e., anticlockwise) cyclic order on the vertices of $\bar{\nu}$ and we set:

$$
\forall t, \gamma_{z}(t)=z+t\left(z^{\prime}-z\right)
$$

- These strings uniquely determine an element of $\Omega\left(X_{n}, \mu_{n}\right)$ which we denote by $\gamma_{\lambda}$.

Lemma 4.2. Let $\lambda \models \mu_{n}$, and let $\zeta=e^{\frac{2 i \pi}{n}}$.

(i) The loop $\gamma_{\lambda}$ represents $\delta_{\lambda}$.

(ii) $\forall z \in \mu_{n}, \gamma_{z \cdot \lambda}=z \cdot \gamma_{\lambda}$.

(iii) Let $\lambda^{\prime}=\mu_{n}$. We have $\lambda=\lambda^{\prime} \Leftrightarrow \delta_{\lambda}=\delta_{\lambda^{\prime}} \Leftrightarrow \gamma_{\lambda}=\gamma_{\lambda^{\prime}}$.

(iv) Let $k \in \mathbf{N}$. Denote by $d$ the order of the root of unity $\zeta^{k}$. Then

$$
\zeta^{k} \lambda=\lambda \Leftrightarrow \delta^{k} \delta_{\lambda}=\delta_{\lambda} \delta^{k} \Leftrightarrow \gamma_{\lambda} \in \Omega\left(X_{n}^{\mu_{d}}, \mu_{n}\right) .
$$

Proof. The first three statements are obvious consequences of the previous definition.

As $\delta^{-k} \delta_{\lambda} \delta^{k}=\delta_{\zeta^{k} \lambda}$, we have, using (iii),

$$
e^{\frac{2 i k \pi}{n}} \lambda=\lambda \Leftrightarrow \delta^{k} \delta_{\lambda}=\delta_{\lambda} \delta^{k} \Leftrightarrow \gamma_{\zeta^{k} \lambda}=\gamma_{\lambda} .
$$

By (ii), we have

$$
\gamma_{\zeta^{k} \lambda}=\gamma_{\lambda} \Leftrightarrow \zeta^{k} \gamma_{\lambda}=\gamma_{\lambda} .
$$

It is clear that $\zeta^{k} \gamma_{\lambda}=\gamma_{\lambda}$ is equivalent to $\gamma_{\lambda} \in \Omega\left(X_{n}^{\mu_{d}}, \mu_{n}\right)$. We have proved (iv).

\section{Proof of the theorem.}

We start with one particular case.

Proposition 5.1. Let $r, d \in \mathbf{N}$. Let $n=d r$. The inclusion $X_{n}^{\mu_{d}} \subset X_{n}$ induces an isomorphism

$$
\pi_{1}\left(X_{n}^{\mu_{d}}, \mu_{n}\right) \stackrel{\sim}{\longrightarrow} \pi_{1}\left(X_{n}, \mu_{n}\right)^{\mu_{d}} .
$$


Proof. The injectivity part of the proposition has been proved in $[\mathbf{B e}]$. Let us prove the surjectivity. Let $\zeta=e^{\frac{2 i \pi}{d}}$. Multiplication by $\zeta$ coincides with conjugating by $\delta^{n / d}$, so, by 3.6 (ii), we know that $\pi_{1}\left(X_{n}, \mu_{n}\right)^{\mu_{d}}$ is generated by the $\delta_{\lambda}$ such that $\zeta \lambda=\lambda$. Using (iv) of Lemma 4.2 , this means that $\gamma_{\lambda} \in \Omega\left(X_{n}^{\mu_{d}}, \mu_{n}\right)$. Thus any such $\delta_{\lambda}$ is in the image of $\pi_{1}\left(X_{n}^{\mu_{d}}, \mu_{n}\right)$. So we have proved that the image of $\pi_{1}\left(X_{n}^{\mu_{d}}, \mu_{n}\right)$ is $\pi_{1}\left(X_{n}, \mu_{n}\right)^{\mu_{d}}$.

We now have to study the other type of regular numbers for $\mathfrak{S}_{n}$. We denote by $\nu_{n}$ the basepoint of $X_{n}$ defined by $\nu_{n}:=\mu_{n-1} \cup\{0\}$.

Proposition 5.2. Let $r, d \in \mathbf{N}$. Let $n=d r+1$. The inclusion $X_{n}^{\mu_{d}} \subset X_{n}$ induces an isomorphism

$$
\pi_{1}\left(X_{n}^{\mu_{d}}, \nu_{n}\right) \stackrel{\sim}{\longrightarrow} \pi_{1}\left(X_{n}, \nu_{n}\right)^{\mu_{d}} .
$$

Proof. For all integer $m$, let us denote by $X_{m}^{*}$ the space of configurations of $m$ points in $\mathbf{C}^{*}$, with its natural topology.

There is a natural inclusion $X_{m}^{*} \subset X_{m}$ and a natural injection $X_{m}^{*} \subset$ $X_{m+1}$ defined by $x \mapsto x \cup\{0\}$. The action of $\mu_{d}$ on $X_{m}$ restricts to an action on $X_{m}^{*}$. Thus we have a commutative diagram of continuous maps:

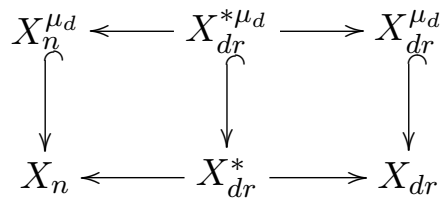

According to $[\mathbf{B e}]$, Lemme 3.1., the first line consists of homeomorphisms. Consider the following commutative diagram of group morphisms:

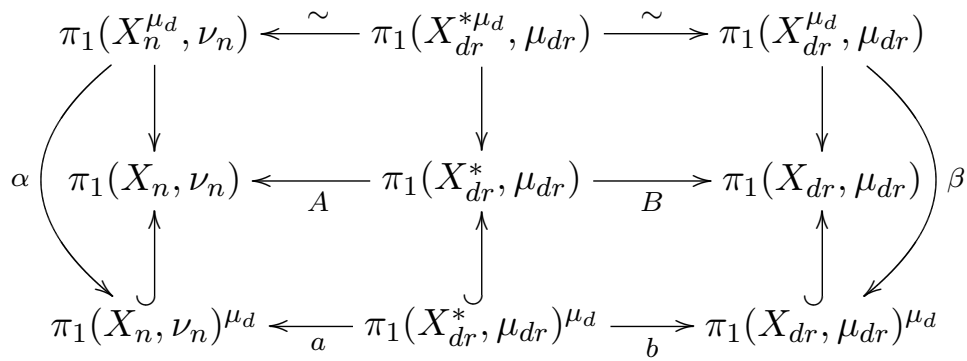

where $\alpha$ and $\beta$ are defined by functoriality, as in the introduction. According to our Proposition 5.1, $\beta$ is an isomorphism. What we want to prove is that $\alpha$ is also an isomorphism. This will result from the fact that both $a$ and $b$ are isomorphisms.

- The map $a$ is an isomorphism: By an easy (and almost classical) argument, one can see that $A$ is injective and identifies $\pi_{1}\left(X_{d r}^{*}, \mu_{d r}\right)$ with the subgroup of $\pi_{1}\left(X_{n}, \nu_{n}\right)$ consisting of elements whose associated 
permutations of $\nu_{n}$ fix the point 0 . As clearly $\pi_{1}\left(X_{n}, \nu_{n}\right)^{\mu_{d}}$ is included in this subgroup, $a$ is an isomorphism.

- The map $b$ is an isomorphism: As noticed in [Be] (fact $(*)$, used in the proof of Theorem 3.2.(I), page 14), there is an exact sequence

$$
1 \longrightarrow F_{n} \longrightarrow \pi_{1}\left(X_{d r}^{*}, \mu_{d r}\right) \underset{B}{\longrightarrow} \pi_{1}\left(X_{d r}, \mu_{d r}\right) \longrightarrow 1
$$

where $F_{n}$ is the free group on $n$ generators and such that the action of $\mu_{d}$ permutes without fixed points the images of the generators of $F_{n}$. As a consequence, the intersection of $\operatorname{ker} B$ with $\pi_{1}\left(X_{d r}^{*}, \mu_{d r}\right)^{\mu_{d}}$ is trivial, and $b$ is injective. The surjectivity of $b$ results from the surjectivity of $B$ and from the $\mu_{d}$-equivariance of the diagram.

Together, the last two propositions prove the part of Theorem 0.2 about symmetric groups, where the only regular numbers are the divisors of $n$ and $n-1$.

Now consider the irreducible reflection group $G(p, 1, n)$, denote by $X_{p, n}$ the complement of its discriminant. As $G(p, 1, n)$ is the centralizer of a $p$ regular element of $\mathfrak{S}_{p n}$, we can identify $X_{p, n}$ and $X_{p n}^{\mu_{p}}$ (in a way compatible with the action of $\mu_{p}$ ).

Let $d$ be a regular number for $G(p, 1, n)$. Consider the following diagram of inclusions:

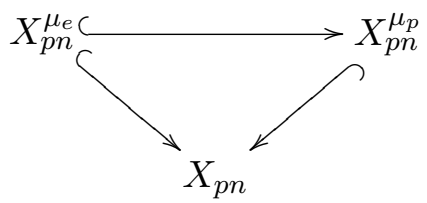

where $e$ is the lcm of $d$ and $p$. By Proposition 5.1, the $\pi_{1}$-images of downward arrows factorize through isomorphisms with centralizers in $\pi_{1}\left(X_{p n}, \mu_{p n}\right)$ (all base points being $\left.\mu_{p n}\right)$ :

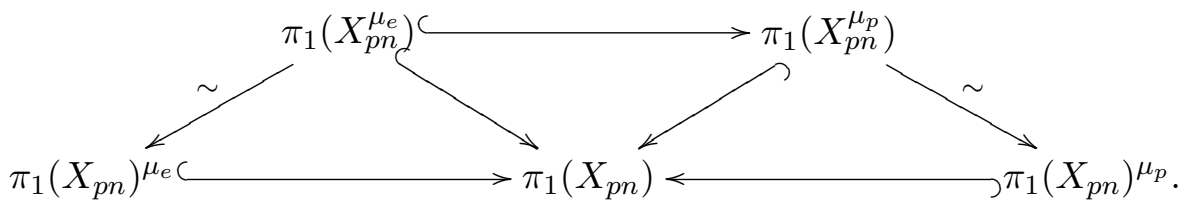

Using the identification between $X_{p, n}$ and $X_{p n}^{\mu_{p}}$, the part of the theorem about $G(p, 1, n)$ says that the natural morphism $\pi_{1}\left(X_{p n}^{\mu_{e}}\right) \rightarrow \pi_{1}\left(X_{p n}^{\mu_{p}}\right)$ induces an isomorphism $\pi_{1}\left(X_{p n}^{\mu_{e}}\right) \stackrel{\sim}{\rightarrow} \pi_{1}\left(X_{p n}^{\mu_{p}}\right)^{\mu_{d}}$; this is obvious on the above diagram, which identifies $\pi_{1}\left(X_{p n}^{\mu_{e}}\right)$ with $\pi_{1}\left(X_{p n}\right)^{\mu_{e}}$ and $\pi_{1}\left(X_{p n}^{\mu_{p}}\right)$ with $\pi_{1}\left(X_{p n}\right)^{\mu_{p}}$.

To complete the proof of the theorem, it is enough to notice that the exceptional groups mentioned are Shephard groups (see [OrSo]) and that their discriminants are isomorphic to ones we have already studied. 


\section{References}

[Be] D. Bessis, Groupes des tresses et éléments réguliers, J. Reine Angew. Math., 518 (2000), 1-40, MR 2001c:20082, Zbl 0961.20033.

[BiKoLee] J. Birman, K.H. Ko and S.J. Lee, A new approach to the word and conjugacy problem in the braid groups, Adv. Math., 139(2) (1998), 322-353, MR 99m:20082, Zbl 0937.20016.

[BrMi] M. Broué and J. Michel, Sur certains éléments réguliers des groupes de Weyl et les variétés de Deligne-Lusztig associées, Proceedings de la Semaine de Luminy 'Représentations des groupes réductifs finis,' 73-139, Birkhaüser, 1996, MR 98h:20077.

[DePa] P. Dehornoy and L. Paris, Gaussian groups and Garside groups, two generalizations of Artin groups, Proc. of London Math. Soc., 79 (1999), 569-604, MR 2001f:20061.

[Mi] J. Michel, A note on words in braid monoids, J. of Algebra, 215 (1999), 366377, MR 2000b:20046, Zbl 0937.20017.

[OrSo] P. Orlik and L. Solomon, Discriminants in the invariant theory of reflection groups, Nagoya Math. J., 109 (1988), 23-45, MR 89i:32025, Zbl 0614.20032.

[Se] V. Sergiescu, Graphes planaires et présentations des groupes de tresses, Math. Z., 214(3) (1993), 477-490, MR 95j:20034, Zbl 0819.20040.

[ShTo] G.C. Shephard and J.A. Todd, Finite unitary reflection groups, Canad. J. Math., 6 (1954), 274-304, MR 15,600b, Zbl 0055.14305.

[Sp] T.A. Springer, Regular elements of finite reflection groups, Invent. Math., 25 (1974), 159-198, MR 50 \#7371, Zbl 0287.20043.

Received October 4, 2000 and revised January 2, 2001.

YALE UNIVERSITY

DEPARTMENT OF MATHEMATiCs

PO Box 208 283, New Haven CT 06520-8283

E-mail address: db278@math.yale.edu

LAMFA, Université de PicARdie-Jules Verne

33, Rue SAint-Leu

80039 Amiens Cedex

FRANCE

E-mail address: digne@u-picardie.fr

LAMFA, Université de Picardie-Jules Verne

33, Rue Saint-Leu

80039 Amiens Cedex

FRANCE

Institut DE MAThÉmATiQUes

Université PARIS VII

175, Rue Du Chevaleret

75013 PARIS

FRANCE

E-mail address: jmichel@math.jussieu.fr 